PROCEEDINGS OF THE

AMERICAN MATHEMATICAL SOCIETY

Volume 136, Number 12, December 2008, Pages 4197-4200

S 0002-9939(08)09628-7

Article electronically published on July 23, 2008

\title{
LINEAR MAPS PRESERVING INVARIANTS
}

\author{
GERALD W. SCHWARZ
}

(Communicated by Gail R. Letzter)

\begin{abstract}
Let $G \subset \mathrm{GL}(V)$ be a complex reductive group. Let $G^{\prime}$ denote $\left\{\varphi \in \operatorname{GL}(V) \mid p \circ \varphi=p\right.$ for all $\left.p \in \mathbb{C}[V]^{G}\right\}$. We show that, "in general", $G^{\prime}=G$. In case $G$ is the adjoint group of a simple Lie algebra $\mathfrak{g}$, we show that $G^{\prime}$ is an order 2 extension of $G$. We also calculate $G^{\prime}$ for all representations of $\mathrm{SL}_{2}$.
\end{abstract}

\section{INTRODUCTION}

Our base field is $\mathbb{C}$, the field of complex numbers. Let $G \subset \mathrm{GL}(V)$ be a reductive group. Let $G^{\prime}=\left\{\varphi \in \operatorname{GL}(V) \mid p \circ \varphi=p\right.$ for all $\left.p \in \mathbb{C}[V]^{G}\right\}$. Several authors have studied the problem of determining $G^{\prime}$. If $G$ is finite, then one easily sees that $G^{\prime}=G$. Solomon [Sol05, Sol06 has classified many triples consisting of reductive groups $H \subset G$ and a $G$-module $V$ such that $\mathbb{C}(V)^{H}=\mathbb{C}(V)^{G}$ (rational invariant functions). If $G$ and $H$ are semisimple, then this is the same thing as finding triples where we have equality of the polynomial invariants: $\mathbb{C}[V]^{H}=\mathbb{C}[V]^{G}$. We show that for "general" faithful $G$-modules $V$ we have that $G=G^{\prime}$. We also compute $G^{\prime}$ for all representations of $\mathrm{SL}_{2}$.

First we study the case that $G$ is the adjoint group of a simple Lie algebra $\mathfrak{g}$. Our interest in this case is due to the paper of Raïs Rai07. where the question of determining $G^{\prime}$ is raised. The case that $\mathfrak{g}=\mathfrak{s l}_{n}$ was also settled by him Rai72, where it is shown that $G^{\prime} / G$ is generated by the mapping $\mathfrak{s l}_{n} \ni X \mapsto X^{t}$ where $X^{t}$ denotes the transpose of $X$. In $\S 2$ we show that, "in general", $G^{\prime} / G$ is generated by the element $-\psi$ where $\psi: \mathfrak{g} \rightarrow \mathfrak{g}$ is a certain automorphism of $\mathfrak{g}$ of order 2 . In the case of $\mathfrak{s l}_{n}, \psi(X)=-X^{t}$, so that our result reproduces that of Raïs. The computation of $G^{\prime}$ for $\mathfrak{g}$ semisimple follows easily from the case that $\mathfrak{g}$ is simple. In $\S 3$ we prove our result that $G=G^{\prime}$ for "general" $G$ and "general" $G$-modules $V$. In $\S 4$ we consider representations of $\mathrm{SL}_{2}$.

We thank M. Raïs for bringing his paper Rai07 to our attention, and we thank him and D. Wehlau for help and advice.

Received by the editors November 14, 2007.

2000 Mathematics Subject Classification. Primary 20G20, 22E46, 22E60.

Key words and phrases. Invariant polynomials.

This work was partially supported by NSA Grant H98230-06-1-0023.

(C)2008 American Mathematical Society Reverts to public domain 28 years from publication 


\section{THE ADJOINT CASE}

Proposition 2.1. Let $\mathfrak{g}$ be a semisimple Lie algebra, so we have $\mathfrak{g}=\mathfrak{g}_{1} \oplus \cdots \oplus \mathfrak{g}_{r}$ where the $\mathfrak{g}_{i}$ are simple ideals. Let $\varphi \in G^{\prime}$. Then $\varphi\left(\mathfrak{g}_{i}\right)=\mathfrak{g}_{i}$ for all $i$, and $\left.\varphi\right|_{\mathfrak{g}_{i}}= \pm \sigma_{i}$ where $\sigma_{i}$ is an automorphism of $\mathfrak{g}_{i}$.

Proof. By a theorem of Dixmier Dix79] we know that the Lie algebra of $G^{\prime}$ is $\operatorname{ad}(\mathfrak{g}) \subset \mathfrak{g l}(\mathfrak{g})$. Thus $\varphi$ acts on $\operatorname{ad}(\mathfrak{g}) \simeq \mathfrak{g}$ via an automorphism $\sigma$ where $\varphi \circ \operatorname{ad} X \circ$ $\varphi^{-1}=\operatorname{ad} \sigma(X)$ for $X \in \mathfrak{g}$. Since $\varphi$ induces the identity on $\mathbb{C}[\mathfrak{g}]^{G}$, so does $\sigma$, and it follows that $\sigma=\prod_{i} \sigma_{i}$ where $\sigma_{i} \in \operatorname{Aut}\left(\mathfrak{g}_{i}\right), i=1, \ldots, r$. By Schur's lemma, $\varphi \circ \sigma^{-1}$ restricted to $\mathfrak{g}_{i}$ is multiplication by some scalar $\lambda_{i} \in \mathbb{C}^{*}, i=1, \ldots, r$. Since $\operatorname{Aut}\left(\mathfrak{g}_{i}\right)$ and $G^{\prime}$ preserve the invariant of degree 2 corresponding to the Killing form on each $\mathfrak{g}_{i}$ we must have that $\lambda_{i}= \pm 1, i=1, \ldots, r$.

From now on we assume that $\mathfrak{g}$ is simple. Let $\sigma \in \operatorname{Aut}(\mathfrak{g})$. Then we know that, up to multiplication by an element of $G=\operatorname{Aut}(\mathfrak{g})^{0}$, we can arrange that $\sigma$ preserves a fixed Cartan subalgebra $\mathfrak{t} \subset \mathfrak{g}$. Thus we may assume that $\varphi$ preserves $\mathfrak{t}$. Let $T$ denote the corresponding maximal torus of $G$.

Corollary 2.2. We may modify $\varphi$ by an element of $G$ so that $\varphi$ is the identity on $\mathfrak{t}$.

Proof. By Chevalley's theorem, restriction to $\mathfrak{t}$ gives an isomorphism of $\mathbb{C}[\mathfrak{g}]^{G}$ with $\mathbb{C}[\mathfrak{t}]^{W}$, where $W$ is the Weyl group of $\mathfrak{g}$. Thus the restriction of $\varphi$ to $\mathfrak{t}$ coincides with an element of $W$, where every element of $W$ is the restriction of an element of $G$ stabilizing $\mathfrak{t}$. Thus we may assume that $\varphi$ is the identity on $\mathfrak{t}$.

Let $\varphi$ be the set of roots and $\varphi^{+}$a choice of positive roots. Let $\Pi$ denote the set of simple roots. Since $\varphi= \pm \sigma$ is the identity on $\mathfrak{t}, \sigma(x)=c_{\sigma} x$ for all $x \in \mathfrak{t}$, where $c_{\sigma}= \pm 1$. Hence either $\sigma$ sends each $\mathfrak{g}_{\alpha}$ to itself or it sends each $\mathfrak{g}_{\alpha}$ to $\mathfrak{g}_{-\alpha}, \alpha \in \varphi$. Choose nonzero elements $x_{\alpha} \in \mathfrak{g}_{\alpha}, \alpha \in \Pi$, and choose elements $y_{\alpha} \in \mathfrak{g}_{-\alpha}$ such that $\left(x_{\alpha}, y_{\alpha},\left[x_{\alpha}, y_{\alpha}\right]\right)$ is an $\mathfrak{s l}_{2}$-triple. Let $\psi$ denote the unique order 2 automorphism of $\mathfrak{g}$ such that $\psi(x)=-x, x \in \mathfrak{t}$ and $\psi\left(x_{\alpha}\right)=-y_{\alpha}, \alpha \in \Pi$ (see [Hum72, 14.3]).

Proposition 2.3. (1) If $c_{\sigma}=1$, then $\sigma$ is inner.

(2) If $c_{\sigma}=-1$, then $\sigma$ differs from $\psi$ by an element of $\operatorname{Ad}(T)$.

Proof. If $c_{\sigma}=1$, then $\sigma\left(x_{\alpha}\right)=c_{\alpha} x_{\alpha}, c_{\alpha} \in \mathbb{C}, \alpha \in \Pi$. There is a $t \in T$ such that $\operatorname{Ad}(t)\left(x_{\alpha}\right)=c_{\alpha} x_{\alpha}, \alpha \in \Pi$. It follows that $\sigma=\operatorname{Ad}(t) \in G$. If $c_{\sigma}=-1$, we can modify $\sigma$ by an element of $T$ so that it becomes $\psi$.

Proposition 2.4. Let $\mathfrak{g}$ be simple. Then the following are equivalent.

(1) Every representation of $\mathfrak{g}$ is self-dual.

(2) The automorphism $\psi$ is inner.

(3) The generators of $\mathbb{C}[\mathfrak{g}]^{G}$ have even degree.

(4) $\mathfrak{g}$ is of the following type:

(a) $\mathrm{B}_{\mathrm{n}}, n \geq 1$,

(b) $\mathrm{C}_{\mathrm{n}}, n \geq 3$,

(c) $\mathrm{D}_{2 \mathrm{n}}, n \geq 2$,

(d) $E_{7}, E_{8}, F_{4}$ or $G_{2}$.

Proof. The equivalence of (10), (3) and (4) is well-known. Now given a highest weight vector $\lambda$ of $\mathfrak{g}$, the highest weight vector of the corresponding dual representation 
$V(\lambda)^{*}$ is $-\rho(\lambda)$, where $\rho$ is the unique element of the Weyl group $W$ which sends $\varphi^{+}$to $\varphi^{-}$([Hum72, $\S 21$, Exercise 6]). Suppose that we have (2). Then, since $\psi$ is inner and it normalizes $\mathfrak{t}$, it gives an element of $W$, namely $\rho$, so that $V(\lambda)^{*} \simeq V(\lambda)$ for all $\lambda$ and (11) holds. Conversely, if (11) holds, then $-\rho$ is the identity on the set of weights; hence $\rho(\alpha)=-\alpha$ for all $\alpha \in \varphi$. It follows that $\rho \circ \psi$ is an automorphism of $\mathfrak{g}$ which is the identity on $\mathfrak{t}$ and sends $\mathfrak{g}_{\alpha}$ to $\mathfrak{g}_{\alpha}$ for all $\alpha$. Then $\rho \circ \psi \in \operatorname{Ad}(T)$ so that $\psi$ is inner.

Theorem 2.5. The group $G^{\prime} / G$ has order 2 , generated by $-\psi$.

Proof. If $\varphi=\sigma \in \operatorname{Aut}(\mathfrak{g})$, then Proposition 2.3 shows that $\varphi=\sigma \in G$. If $\varphi=$ $-\sigma$, then by Proposition 2.3 we may assume that $\varphi=-\psi$. Now $-\psi$ induces an automorphism of $\mathbb{C}[\mathfrak{g}]^{G}$ and $-\psi$ is the identity on $\mathfrak{t}$. Hence Chevalley's theorem shows that $-\psi \in G^{\prime}$, and we know that $-\psi$ generates $G^{\prime} / G$. Moreover, $-\psi$ is not in $\operatorname{Aut}(\mathfrak{g})$, so that $-\psi \notin G$.

Corollary 2.6. Suppose that $\psi$ is inner. Then $G^{\prime} / G$ is generated by multiplication by -1 .

We leave it to the reader to formulate versions of Theorem 2.5 and Corollary 2.6 for the semisimple case.

\section{The General CASE}

We have a finite dimensional vector space $V$ and $G$ is a reductive subgroup of $\operatorname{GL}(V)$. Let $G^{\prime}:=\left\{\varphi \in \operatorname{GL}(V) \mid p \circ \varphi=p\right.$ for all $\left.p \in \mathbb{C}[V]^{G}\right\}$. We show that, "in general", we have $G^{\prime}=G$.

Let $U$ denote the subset of $V$ consisting of closed $G$-orbits with trivial stabilizer. It follows from Luna's slice theorem [Lun73] that $U$ is open in $V$.

Theorem 3.1. Suppose that $V \backslash U$ is of codimension 2 in $V$. Then $G^{\prime}=G$.

Proof. Let $\varphi \in G^{\prime}$ and let $x \in U$. Then $\varphi(x)=\psi(x) \cdot x$ where $\psi: U \rightarrow G$ is a well-defined morphism. Since $G$ is affine, we may consider $\psi$ as a mapping from $U \rightarrow G \subset \mathbb{C}^{n}$ for some $n$ where $G$ is Zariski closed in $\mathbb{C}^{n}$. Our condition on the codimension of $V \backslash U$ guarantees that each component of $\psi$ is a regular function on $V$; hence $\psi$ extends to a morphism defined on all of $V$, with image in $G$. Now let $x \in U$. Then

$$
\varphi(x)=\lim _{t \rightarrow 0} \varphi(t x) / t=\lim _{t \rightarrow 0} \psi(t x) t x / t=\psi(0)(x) .
$$

Thus $\varphi$ is just the action of $\psi(0) \in G$, so $G^{\prime}=G$.

\section{Representations OF $\mathrm{SL}_{2}$}

As an illustration, we consider representations of $G=\mathrm{SL}_{2}$ or $G=\mathrm{SO}_{3}$. We only consider representations with no nonzero fixed subspace. We let $R_{j}$ denote the irreducible representation of dimension $j+1, j \geq 0$, and $k R_{j}$ denotes the direct sum of $k$ copies of $R_{j}, k \geq 1$. When we have a representation only containing copies of $R_{j}$ for $j$ even, then we are considering representations of $\mathrm{SO}_{3}$. From [Sch95, 11.9] we know that all representations of $G$ satisfy the hypotheses of Theorem 3.1 except for the following cases, where we compute $G^{\prime}$.

(1) For $R_{1}$ we have $G^{\prime}=\mathrm{GL}_{2}$, for $2 R_{1}$ we have $G^{\prime}=\mathrm{O}_{4}$ and for $3 R_{1}$ we have $G^{\prime}=G$. 
(2) For $R_{2}$ we have $G^{\prime}=\mathrm{O}_{3}$ and for $2 R_{2}$ we have $G^{\prime}=\mathrm{O}_{3}$. (Here $G=\mathrm{SO}_{3}$.)

(3) For $R_{2} \oplus R_{1}$ we have $G^{\prime}=\left\{g^{\prime} \in \mathrm{GL}_{2} \mid \operatorname{det}\left(g^{\prime}\right)= \pm 1\right\}$.

(4) For $R_{3}$ the group $G^{\prime}$ is the same as in case (3).

(5) For $R_{4}$ we have $G^{\prime}=G=\mathrm{SO}_{3}$.

Most of the calculations are easy. We mention some details for some of the nonobvious cases.

Suppose that our representation is $R_{4}$, which has generating invariants of degrees 2 and 3. The Lie algebra $\mathfrak{g}^{\prime}$ acts irreducibly on $R_{4}$; hence it is the sum of a center and a semisimple Lie algebra ([Jac62, Ch. II, Theorem 11]). Clearly we cannot have a nontrivial center, so that $\mathfrak{g}^{\prime}$ is semisimple. Now a case-by-case check of the possibilities forces $\mathfrak{g}^{\prime}=\mathfrak{g}$. Suppose that $g^{\prime} \in G^{\prime} \backslash G$. Then conjugation by $g^{\prime}$ gives an inner automorphism of $G$; hence we can correct $g^{\prime}$ by an element of $G$ so that $g^{\prime}$ commutes with $G$. Thus $g^{\prime}$ acts on $R_{4}$ as a scalar. But to preserve the invariants the scalar must be 1 . Thus we have $G^{\prime}=G$. Similar considerations give that $\mathfrak{g}^{\prime}=\mathfrak{g}$ in case (4), so that $G^{\prime} / G$ is generated by scalar multiplication by $i$ (since the generating invariant of $R_{3}$ has degree 4 ), which shows that $G^{\prime}$ is as claimed.

In case (3), one sees that $\mathfrak{g}^{\prime}=\mathfrak{g}$, so that generators of $G^{\prime} / G$ act as scalars on $R_{2}$ and $R_{1}$. Now generators of the invariants have degrees $(2,0)$ and $(1,2)$ so that $G^{\prime} / G$ is generated by an element which is multiplication by -1 on $R_{2}$ and multiplication by $i$ on $R_{1}$. Hence $G^{\prime}$ is as claimed.

\section{REFERENCES}

[Dix79] J. Dixmier, Champs de vecteurs adjoints sur les groupes et algèbres de Lie semisimples, J. Reine Angew. Math. 309 (1979), 183-190. MR542047 (80i:17011)

[Hum72] J.E. Humphreys, Introduction to Lie Algebras and Representation Theory, Graduate Texts in Mathematics 9, Springer-Verlag, New York, 1972. MR499562 (81b:17007)

[Jac62] N. Jacobson, Lie Algebras, Interscience Publishers, New York, 1962. MR0143793 $(26: 1345)$

[Lun73] D. Luna, Slices étales, Bull. Soc. Math. France, Mémoire 33 (1973), 81-105. MR0342523 (49:7269)

[Rai72] M. Raïs, Distributions homogènes sur des espaces de matrices, Bull. Soc. Math. France, Mémoire 30 (1972). MR0507255 (58:22412)

[Rai07] Notes sur la notion d'invariant caractéristique, http://arxiv.org/abs/0707. $0782 \mathrm{v} 1$.

[Sch95] G.W. Schwarz, Lifting differential operators from orbit spaces, Ann. Sci. Ecole Norm. Sup. 28 (1995), 253-306. MR:1326669 (96f:14061)

[Sol05] S. Solomon, Irreducible linear group-subgroup pairs with the same invariants, J. Lie Theory 15 (2005), no. 1, 105-123. MR2115231 (2005j:13008)

[Sol06] Orthogonal linear group-subgroup pairs with the same invariants, J. of Alg. 299 (2006), no. 2, 623-647. MR2228331 (2007c:13007)

Department of Mathematics, Brandeis University, Waltham, Massachusetts 024549110

E-mail address: schwarz@brandeis.edu 